\title{
Zur gegenwärtigen Bedeutung des Körpersignals Bart für die Kommunikation
}

C. Wietig

\author{
To the Current Relevance of the Aesthetical Body-Image Concerning Beard- \\ Styling as Virile Expression for Visual Communication - "Bodystyling - How Much \\ Beard Initiates Being a Man? Questionnaire-Catalogue for Men 2003”
}

Zusammenfassung

Hintergrund: Die Zahl der Bartträger ist nach bisherigen Untersuchungen gegenüber den Nichtbartträgern seit der Entwicklung der Selbstrasur und auch seit dem Zweiten Weltkrieg deutlich gesunken. Ziel der vorliegenden Untersuchung war daher, die derzeitige Bedeutung des männlichen Bartes für die Kommunikation innerhalb der deutschen Kultur zu evaluieren.

Methodik: Mithilfe einer nicht randomisierten, nicht repräsentativen Datenerhebung wurde eine deskriptive Analyse „Bodystyling - Wie viel Bart braucht der Mann? Fragenkatalog zum Bart für Männer 2003“ sowie einer subsumierten Printmedienanalyse von Männern mit Bart in der Werbung aktueller Zeitschriften und Magazine durchgeführt. Das Körperfragment Bart wurde als pars pro toto für die Kommunikation analysiert und vor dem Hintergrund kulturwissenschaftlicher Aspekte der Bartphänomenologie diskutiert.

Ergebnisse: Von 524 Männern der Untersuchungsgesamtheit waren 186 Personen Bartträger (35,5\%). Dieses Ergebnis stimmte etwa mit einer repräsentativen Datenerhebung aus dem Jahr 1990 überein. Die Ergebnisse deuten an, dass etwa von einem Drittel der Männer die Kontinuität einen Bart zu tragen, gewahrt wird und für die Kommunikation von Bedeutung zu sein scheint. $77 \%$ der Bartträger gaben an, ihre Identität durch den Bart zu unterstreichen. Von 38\% aller Befragten der deskriptiven Analyse wurde als attraktivste Bartform der Dreitagebart gewählt. Dieser wurde in der Printmedienanalyse bestätigt.

Schlussfolgerungen: Der Bart scheint für die visuelle Kommunikation körperbildästhetische Rollenentwürfe zu vermitteln und vermag scheinbar subjektive Entwürfe authentischer individueller Persönlichkeitsprofile zu visualisieren und damit die virile Darstellungskompetenz zu unterstützen.
Abstract

Background: Since the development of self-shaving and also since the Second World War the number of men wearing a beard decreased in opposite to shaved men based on previous studies. The aim of the study was to analyse the current relevance of the male beard with regard to communication concerning social lifestyle-concepts within the German cultural scene.

Methods: Based on a descriptive analysis "Bodystyling - How much beard initiates being a man? Questionnaire-catalogue for men 2003" and a subordinate print media analysis of bearded men in advertising of the current press the body-fragment beard as pars pro toto was analysed with reference to communication on the background of cultural scientific aspects of beard-phenotypes.

Results: From 524 men of the determined data of the general study 186 wear a beard (35,5\%). These data correspond nearly with data of a representative study from 1990. The results indicate, that a third part of all men within a community continuously prefer wearing a beard. $77 \%$ of bearded men asserted the support of their identity with beard-styling. From all questioned men of the descriptive analysis $38 \%$ chose the Three-Day-Beard as most attractive beard-fashion. This result was confirmed with the preference of the Three-Day-Beard of the current press analysis.

Conclusions: The analysis of the determined data of the general study confirms that the modified beard-growth represents aesthetical-social role-plays and may contribute to decisive attractiveness of an authentic individual person of character. The results indicate that the fashionable-styled beard seems to support the virile self-realization relating to identity and image. 
Einleitung

In jüngster Zeit wird die Dermatologie neben der medizinischen Hautbehandlung und Hautpflege mit ästhetischen und den Körper verbessernden Fragestellungen konfrontiert, weil diesen zunehmend mehr Aufmerksamkeit in der Gesellschaft gewidmet wird. Die ästhetischen Fragen berühren soziologische, psychologische Hintergründe und Schönheitsideale im modischen Wandel und auch den kulturtechnischen Umgang mit der Haut. Markante Beispiele hierfür sind die seit den 90er Jahren beliebter werdenden Tätowierungen und Piercings [1]. Weitere vermehrte Beachtung finden die Barttrachten in so genannten Lifestyle-Magazinen, die bisher wissenschaftlich relativ wenig in der Literatur reflektiert sind und die körperästhetische Trends spiegeln. Das Ziel dieser Untersuchung war daher, mithilfe eines wissenschaftlich ausgewerteten Fragenkatalogs die Relevanz des Körpersignals Bart als pars pro toto in seiner Bedeutung für die Kommunikation zu evaluieren, darzustellen und zu diskutieren.

Die soziale Bedeutung eines gesunden und formschönen Haupthaares ist gemeinhin bekannt [2]. Welche gesellschaftliche Relevanz hat jedoch das Körperfragment Bart innerhalb des deutschen Kulturkreises für die interindividuelle Kommunikation? Nach bisherigen Untersuchungen ist die Zahl der Bartträger gegenüber den Nichtbartträgern seit der Entwicklung der Selbstrasur und auch seit dem Zweiten Weltkrieg deutlich gesunken.

Hierzu wurde mithilfe einer nicht randomisierten, nicht repräsentativen Datenerhebung eine deskriptive Analyse „Bodystyling - Wie viel Bart braucht der Mann? Fragenkatalog zum Bart für Männer 2003“ die Rolle des Bartes in der lifestyleorientierten Gesellschaft im Zeitraum vom 8.1.-31.8.2003 analysiert und vor dem Hintergrund kulturwissenschaftlicher Aspekte der Bartphänomenologie diskutiert. Subsumierend wurde eine Printmedienanalyse von Männern mit Bart in der Werbung aktueller Zeitschriften und Magazine auf ihre Relevanz hin für die Kommunikation untersucht. Daraus ergibt sich der methodische Aufbau dieser Abhandlung für die Abschnitte Methode, Ergebnisse und Diskussion, die jeweils den Teil der deskriptiven Datenanalyse (I) und den der subsumierten Printmedienanalyse (II) enthalten.

\section{Methode}

Die Methode der deskriptiven Datenerhebung „Bodystyling Wie viel Bart braucht der Mann? Fragenkatalog zum Bart für Männer 2003“ (I) wurde modifiziert, indem bei der Stichprobenauswahl der Untersuchungsgesamtheit auf ein randomisiertes Verfahren verzichtet wurde [3]. Bedingt durch diese Einschränkung lässt die darum nicht repräsentative Statistik keinen Anspruch auf Verallgemeinerung zu. Bei der Analyse kam eine wissenschaftlich abgeklärte Befragungsmethodik zur Anwendung, die sich in der Fragestellung an derjenigen der Deutschen Studienstiftung „Bodycheck - Wie viel Körper braucht der Mensch?“ von 2000 orientierte [4]. Da der männliche Bart gleichermaßen phänomenologischen Signalwert zwischen evolutionsbiologischem Haarwachstum, individuell-subjektiver Gestaltung und kulturkreisgebundener Konvention vermittelt, wurde auf eine eindimensionale, einschränkende Hypothese verzichtet.
Die Personengruppen der Stichproben waren Bundeswehrangehörige, Bartclubmitglieder, Altenheimbewohner, Friseure, Polizisten, Lehrer und Schüler von Staatlichen Gewerbeschulen (Werft und Hafen, Arbeits- und Werktechnik, Bautechnik, Maschinenbau, Installationstechnik, Fertigungs- und Flugzeugtechnik) und von einer Staatlichen Handelsschule sowie Studenten der Universität Hamburg.

Subsumierend wurde eine Analyse „Printmedienanalyse - Männer mit Bart in der Werbung aktueller Zeitschriften und Magazine“ (II) vorgenommen, weil die Printmedien gesellschaftliche Trends, Modeideale, Images und teleologisch merkantilistische Lifestyle-Konzepte spiegeln und somit die derzeitige Bartoptik innerhalb der visuellen Kommunikation dokumentieren. Das Ziel dieser im Zeitrahmen vom 1.7.-29.9.2003 beigeordneten Untersuchung, die keinen Anspruch auf Verallgemeinerung hat, war, zu überprüfen, ob sich Übereinstimmungen zwischen den deskriptiv bevorzugten Bartformen der Untersuchungsgesamtheit und den abgebildeten in den Printmedien finden lassen. Die Auswahl der analysierten Quellen wurde anhand der meist verkauften Auflagen allgemeiner sowie Körperkultur und Lifestyle betreffender Printmedien mithilfe des VDZ-Auflagendienstes (Verband Deutscher Zeitschriftenhändler) getroffen.

Ergebnisse

Darstellung der gesammelten Daten im Hinblick auf die gegenwärtige Bedeutung des Körpersignals Bart für die Kommunikation „Bodystyling - Wie viel Bart braucht der Mann? Fragenkatalog zum Bart für Männer 2003“ (I)

Für die nachfolgende Interpretation der spezifischen Quelldaten sei für die Untersuchungsgesamtheit vorausgesetzt, dass sich durch die Stichprobenauswahl unterschiedliche Verteilungen bezüglich der beruflichen Gruppen und der Alterszuordnungen ergaben, so dass dadurch im Datensatz der Erhebung prozentual verteilte Schieflagen entstanden, die jedoch eine immanente Interpretation zulassen, aber eine allgemeingültige aus oben genannten Gründen nicht erlauben. Im Rahmen der Datenerhebung wurden 524 Männer der Untersuchungsgesamtheit mit gültigen Werten befragt. Hiervon waren 186 Personen Bartträger (35,5\%), wobei dies etwa dem Ergebnis einer repräsentativen Erhebung aus dem Jahr 1990 entsprach [5]. 326 Befragte waren Nichtbartträger und 12 Personen gelegentlich Bart- bzw. Nichtbartträger, die bei den detaillierten Analysen ausgeschlossen wurden.

Am stärksten von 524 Befragten der Untersuchungsgesamtheit mit gültigen Werten vertreten sind $113 \mathrm{BW}$-Soldaten mit $21 \%$, gefolgt von 88 Personen der Staatlichen Gewerbe- und Fachoberschule Bautechnik, die 16,8\% ausmachen, diesen fast gleichberechtigt folgen 83 Personen mit 15,8\% der Staatlichen Gewerbeschule Fertigungs- und Flugzeugtechnik. Die Staatliche Gewerbe- und Fachoberschule Maschinenbau ist mit 47 Personen, die 9,0\% ausmachen, vertreten und der Berliner Bartclub 1996 e.V. stellt mit 34 Personen, die 6,5\% ergeben, neben den noch verbleibenden rangskalierten Restgruppen die stärkste Gruppe dar. 
Von 524 Befragten der Untersuchungsgesamtheit mit gültigen Werten sind: 369 Personen mit 70,4\% 16-29 Jahre, 80 Personen mit 15,3\% 30-44 Jahre, 48 Personen mit 9,2\% 45 - 59 Jahre, 27 Personen mit 5,2\% 60 Jahre und älter.

Fast die Hälfte (45,8\%) aller Befragten unter Berücksichtigung fehlender Angaben von 13 Personen besitzen die höhere Schulbildung, etwas weniger als ein Drittel die der Volksschule und etwa ein Viertel die der Hochschule.

Gut die Hälfte der Befragten (55,9\%) mit Ausnahme 5 fehlender Werte gehört dem Christentum an, 36,6\% gehören keiner Religionsgemeinschaft an. Die restlichen 6,9\% stehen stellvertretend für den Islam oder andere Religionsgemeinschaften.

Die folgende Auswahl der grafischen Darstellung der ermittelten Werte in der hier stark reduzierten Überschau visualisiert gemäß der kategorisierten Fragenkomplexe der deskriptiven Umfrage „Bodystyling - Wie viel Bart braucht der Mann? Fragenkatalog zum Bart für Männer 2003“ (I) das ermittelte Meinungsbild der Befragten der Untersuchungsgesamtheit.

Darstellung der ermittelten Abbildungen von Männern mit Bart in der Werbung im Hinblick auf die gegenwärtige Bedeutung des Körpersignals Bart für die Kommunikation (II)

Es wurde in den Werbeanzeigen der untersuchten Printmedien 493fache Bartoptik ermittelt, wovon 129 den Dreitagebart abbilden. Bei einer durchschnittlichen Gesamtzahl von 9236 Werbeanzeigen beträgt der Anteil der Werbeanzeigen von Männern mit Bart abzüglich der ermittelten Babypersiflagen 5,24\%. Von 129 untersuchten Zeitschriften und Magazinen weisen 27 keine Werbeanzeigen von Männern mit Bart auf. Rangskaliert führt deutlich der Dreitagebart unter den ermittelten abgebildeten Bartkategorien (Abb.1).
Diskussion

Interpretation der Datenanalyse im Hinblick auf die gegenwärtige Bedeutung des Körpersignals Bart für die Kommunikation „Bodystyling - Wie viel Bart braucht der Mann? Fragenkatalog zum Bart für Männer 2003“ (I)

\section{Fragenkomplex Persönliche Einstellung zum Bart von Bartträgern und Nichtbartträgern (Abb. 2 u.3) Bart und Individualität}

77\% der Bartträger sind der Meinung, dass der Bart die Individualität unterstreiche. Die Erfahrungswerte bezüglich des interpersonellen Feedbacks der Betroffenen unterstreichen damit, dass der Bart für den Individualausdruck von Bedeutung ist. Demnach scheint das Körpersignal Bart den physiognomischen Code seines Trägers innerhalb der visuellen Kommunikation zu erweitern und Aufmerksamkeit zu erzeugen. Neben der genetischen Einzigartigkeit bezüglich der Terminalhaardichte, -struktur, -farbe und der Begrenzung der Regio barbae, die für den Individualausdruck des biologischen Körpers verantwortlich ist, vermittelt der Körper als symbolbesetzter Kulturträger sowohl biologisch als auch kulturell überformt Individualität im sozialen Raum [6-7,12].

Die hohe Resonanz der Bartträger in der Beantwortung dieser Frage lässt auf die interkommunikative gegenseitig bestärkende Rückversicherung schließen.

\section{Bart und Blickkontakte}

47\% der Bartträger bestätigen, als Bartträger stärkeren Blickkontakten ausgesetzt zu sein. In der interkommunikativen Auseinandersetzung wird das individuell inszenierte mediale Körperbild in der körpereigenen Bilderzeugung gespiegelt und nach kollektiven, persönlichen und zeitimmanenten Mustern gefiltert [8-10,29]. Da die Wahrnehmung (von griechisch aisthesis, Ästhetik) an den Körper gebunden ist, wird auch das Körpersignal Bart reflexiv erfahren und somit Mechanismen der ästhetischen Verfeinerung, Anpassung oder Verweigerung zugänglich

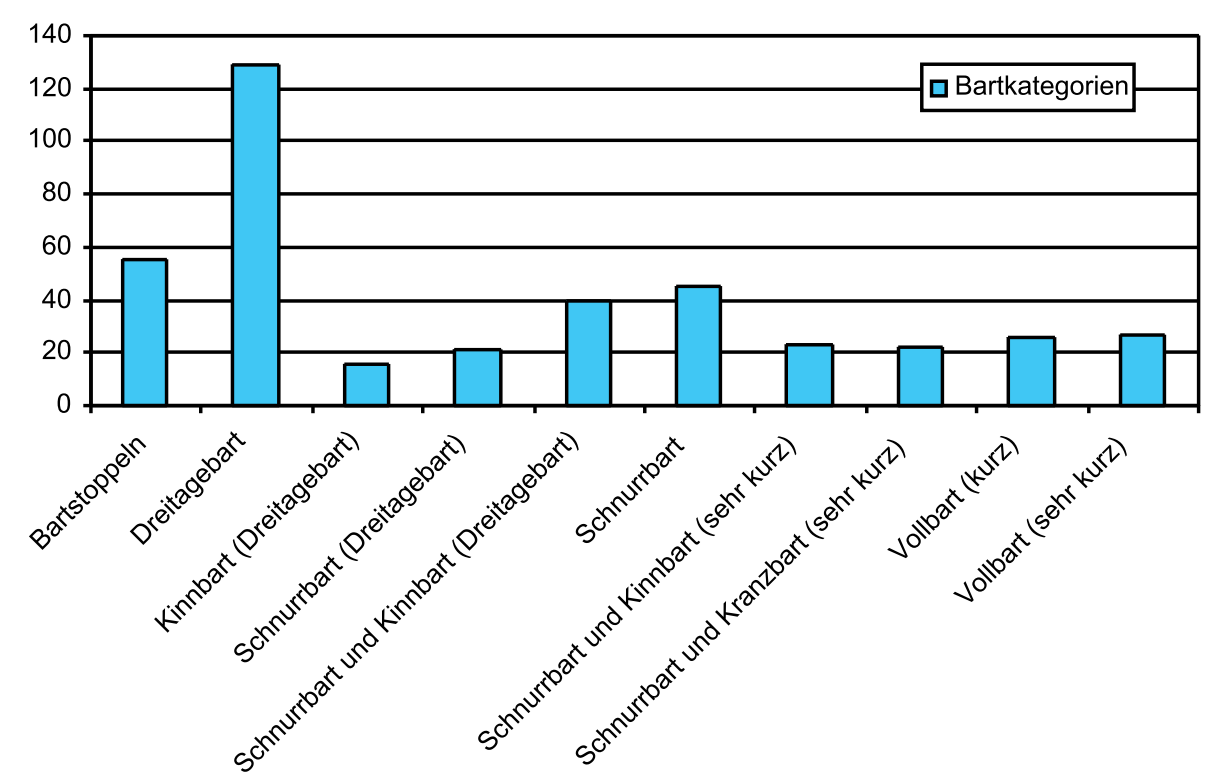

Abb. 1 Anzahl der zahlenmäßig am stärksten vertretenen abgebildeten Bartkategorien in den Werbeanzeigen der Printmedien. 


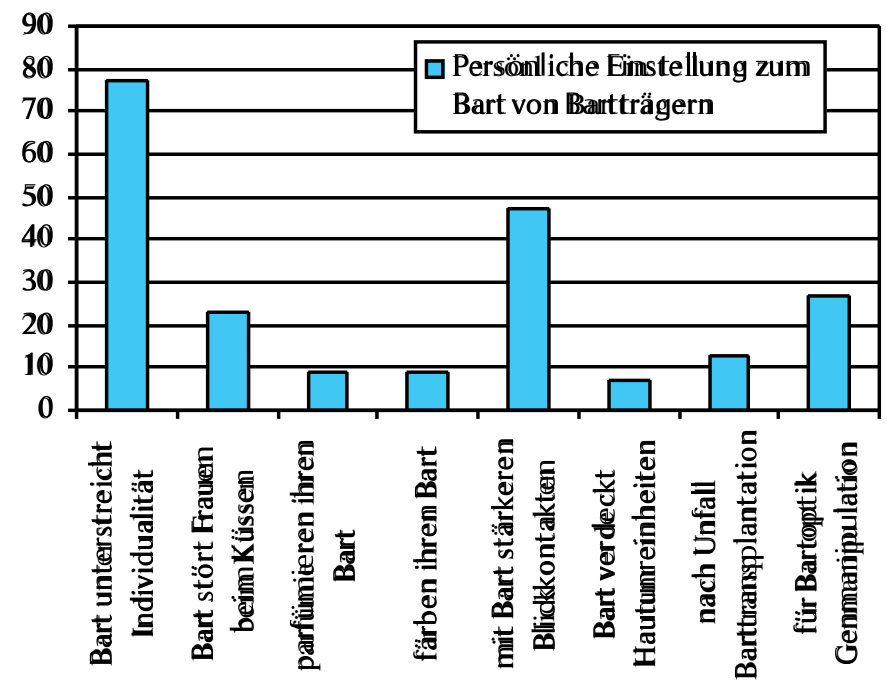

Abb. 2 Persönliche Einstellung zum Bart von Bartträgern.

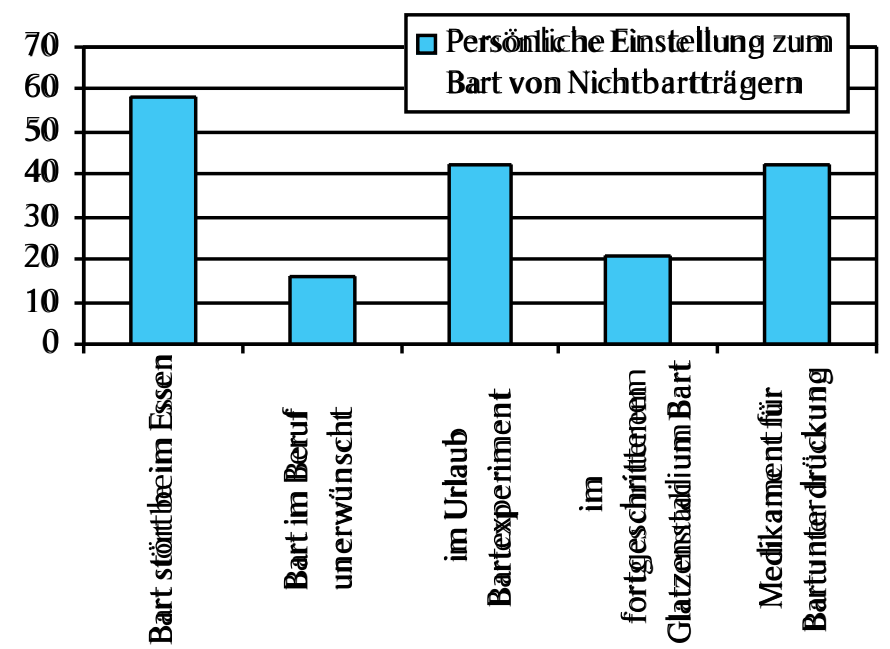

Abb. 3 Persönliche Einstellung zum Bart von Nichtbartträgern.

gemacht $[11,26]$. Das Ergebnis deutet an, dass Bartoptik sowohl eine erweiternde Funktion für die Physiognomik hat als auch für die Kommunikation von Bedeutung ist.

\section{Bartoptik und Genmanipulation}

$27 \%$ der Bartträger wären bereit, Genmerkmale für eine vermeintlich ästhetische Verbesserung der Bartoptik manipulieren zu lassen. Auf der Suche nach dem immer perfekteren Körperbild scheint sich das traditionelle Körperbild nicht nur in der Kunst, sondern auch durch den naturwissenschaftlichen Fortschritt zu dekonstruieren, um sich technisch-prothetisch mithilfe der Erkenntnisse der Biologie, ästhetischen Chirurgie, Genetik und Neurowissenschaft verbessert zu synthetisieren [12-15]. Diesbezüglich scheinen die befürwortenden Bartträger medienorientiert zeitgeistgemäßen Trends gegenüber offen zu sein. Anders ausgedrückt bezeugt diese Zustimmung den hohen sozialen Stellenwert ästhetischer Körperoptik und den noch derzeitigen Aufwand der Pflege für die Form- und Farbgebung. Hierin drückt sich der zunehmende Wunsch aus, die körperliche Präsenz selbst bestimmt konstruiert medial ästhetisch zu inszenieren.

\section{Bartunterdrückung}

$42 \%$ der Nichtbartträger wären bereit, ein Medikament für die Bartunterdrückung einzunehmen. Die Metaphorik des starken Körpers ist eng mit Gesundheit und traditionell mit der Funktionsfähigkeit für die Industriearbeit verbunden. Arbeitsphysiologisch wurde der Leib ab 1860 wissenschaftlich als ökonomisch verwertbarer Muskelapparat mit berechenbarem Energieverbrauch pro Zeiteinheit für die kapitalistische Produktionsweise erforscht und fortschrittsorientiert eingesetzt. Dieser Entwicklung zufolge bedeutet auch heute noch Zeit Geld [16]. Die medizinischen Leistungen und innovativen Techniken der kosmetischen Chirurgie vermögen heute die soziale und kulturelle Identität des Körpers in der modernen Leistungs- und Freizeitgesellschaft neu zu besetzen [17].

\section{Fragenkomplex Bart und Attraktivität (Abb. 4) Bart und Potenz}

Üppiger Haar- und Bartwuchs werden evolutionsbiologisch, urmagisch und soziokulturell mit Dominanz und vermeintlicher Potenz gleichgesetzt [18]. Bis heute scheint die Magie der Kraft des Haares an nichts eingebüßt zu haben [19-21]. Alle zustimmenden Befragten bis auf die 30-44-jährigen Nichtbartträger assoziieren starken Bartwuchs mit gesteigerter Potenz. Wie oben erwähnt besitzen fast die Hälfte aller Befragten unter Berücksichtigung fehlender Angaben von 13 Personen die höhere Schulbildung, etwas weniger als ein Drittel die der Volksschule und etwa ein Viertel die der Hochschule. Scheinbar beurteilen die zustimmenden Befragten der Untersuchungsgesamtheit, wenn auch unterschiedlich gewichtet, den Bartwuchs nicht emotionsfrei und darum nicht objektiv.

\section{Fragenkomplex Bartträger und Image (Abb. 5 u. Abb. 6) Bart und Unkonventionalität}

67\% der 60-jährigen und älteren Bartträger bestätigen, dass ein Dreitagebart Unkonventionalität signalisiere, rangskaliert gefolgt von fast der Hälfte der 30-44-jährigen Bartträger (48\%). $36 \%$ der 16-29-jährigen Bartträger bejahen diese Feststellung sowie $31 \%$ der 45 - 59-jährigen Bartträger. Durchschnittlich $42 \%$ der Nichtbartträger aller Altersstufen stimmen dieser zu, womit sich eine relativ übereinstimmende Meinung aller Altersstufen der Untersuchungsgesamtheit spiegelt. Die Rasur stellt kommunikativ eine Beschwichtigungsgeste dar [22]. Typologisch bietet der Dreitagebart in Abhängigkeit von Persönlichkeitsmerkmalen

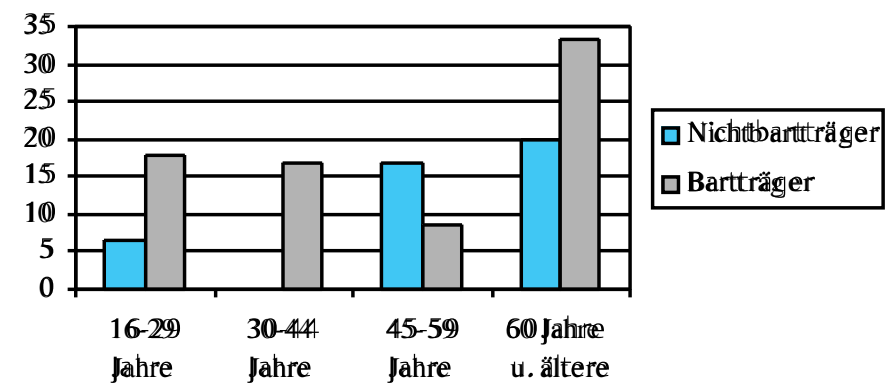

Abb. 4 Zeugt ein starker Bartwuchs von verstärkter Potenz? 


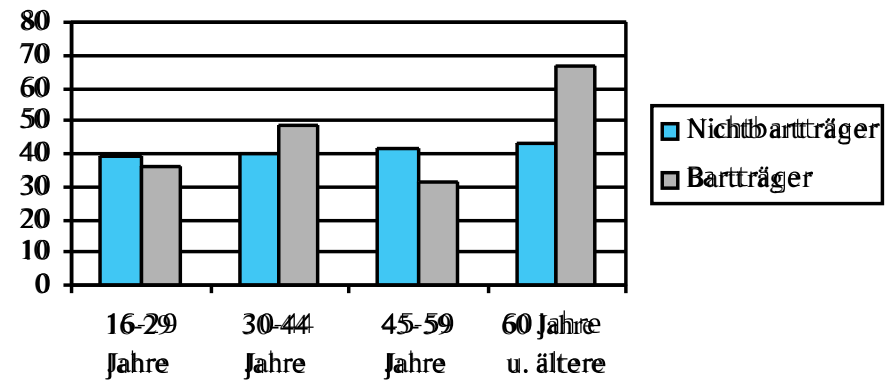

Abb. 5 Ein Dreitagebart signalisiert Unkonventionalität.

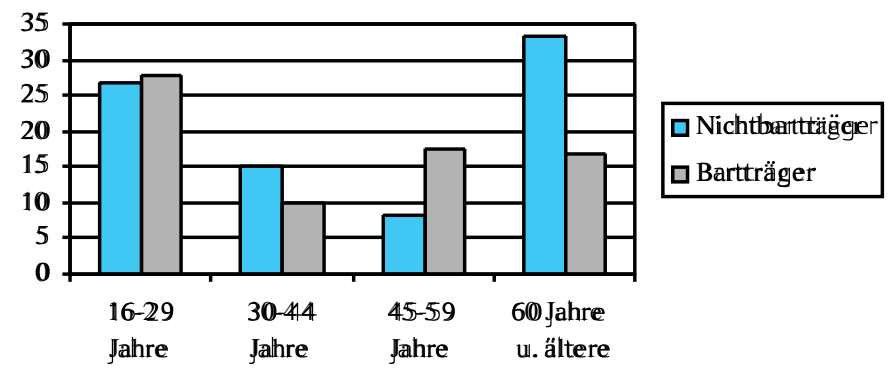

Abb. 6 Lässt ein Bart auf das soziale Milieu des Trägers rückschließen?

einen variantenreichen Spielraum für die Kommunikation. Er kann unter anderem einen anpassungsunwilligen Abenteurer, einen eigenwilligen Individualisten oder einen trendorientierten modebewussten Dandy symbolisieren $[23,10]$. Die untersuchten Printmedien des Jahres 2003 geben den Trend zum bevorzugten Dreitagebart in denjenigen sozialen Milieus wieder, die unabhängig von Einschränkungen modische Selbstverwirklichungen zulassen. Die Ergebnisse dieser Untersuchung deuten an, dass das Körpersignal Dreitagebart von den Befragten relativ deutlich als Zeichen der Unkonventionalität gesehen wird.

\section{Bart und Sozialstatus}

Die Übereinstimmung von durchschnittlich $28 \%$ der 16-29-jährigen Bartträger und Nichtbartträger in der Einschätzung des sozialen Milieus scheint anzudeuten, dass das Körpersignal Bart als pars pro toto für die Kommunikation bezüglich der Einschätzung sozialer Milieus für die Befragten dieser Altersstufe von Bedeutung ist. Soziale Milieus spiegeln gleichermaßen ästhetische Dimensionen des Lebensstils und dienen der Orientierung für die Ausformung des angemessenen Körperkults [24]. Jedes soziale Milieu verfügt daher über entsprechende Wertorientierungen und ästhetische Kompetenz auf der Ebene des individuellen Geschmacks [25]. Die verfeinerte Ästhetisierung der Regio barbae kann daher charakteristische Merkmale ausdrücken und unter Berücksichtigung des Gesamteindruckes Rückschlüsse auf das soziale Milieu zulassen. Jeder zehnte der 30-44-jährigen Bartträger und jeder sechste der 45-59-jährigen Bartträger vermag das soziale Milieu auszumachen. Vermutlich charakterisiert dieses kritischere Ergebnis die vorsichtigere und/oder tolerantere Sichtweise bezüglich der Einschätzung des sozialen Milieus anhand der Interpretation der Bartästhetik.
Fragenkomplex Bart und Charakter (Abb. 7)

\section{Bart und Charakter}

Der Charakter, griechisch „eingeritztes Zeichen“, vermittelt die bleibende Eigenart eines Menschen, seine geistig-sittliche Wesensart, wie er sich als Persönlichkeit fühlend und handelnd in Willensentscheidungen, Wertungen und Zielsetzungen mit der Gesellschaft auseinandersetzt. In Bezug auf die modische Selbstdarstellung bedeutet dies im Technologiezeitalter der ästhetisierten Bodycodes, akzentuiert einzigartige Zeichen im Detail zu setzen. Das relativ konforme Ergebnis der Analyse in Bezug auf die Zustimmung der jeweiligen Altersstufen legt nahe, dass individuell konstruierte Bartästhetik deutliche Botschaften für die Kommunikation zu vermitteln scheint, die Rückschlüsse auf die Persönlichkeitsmerkmale ihres Trägers zulassen. Typenpsychologisch scheinen ästhetische Modifikationen individueller Einzelmerkmale wie die des Bartes Persönlichkeitsstrukturen zu charakterisieren. Die Typologie der Persönlichkeitspsychologie steht in der Tradition der Physiognomik und Temperamentenlehre und lässt darum aufgrund ihrer Pseudowissenschaftlichkeit keinen Anspruch auf Verallgemeinerung zu $[26,18]$.

\section{Fragenkomplex Bart und Weltanschauung (Abb. 8) Bart und Religion}

Ein auffälliger Unterschied in der Bewertung der Frage, ob lange Bärte die Zugehörigkeit zu einer Religionsgemeinschaft ausdrückten, ist bei den 60-jährigen und älteren Bartträgern (17\%) und Nichtbartträgern (53\%) derselben Altersstufe sowie bei den 45-59-jährigen Bartträgern (11\%) und Nichtbartträgern (25\%) auszumachen. Die prozentual geringere Zustimmung der Bartträger deutet an, dass diese die Frage entweder aufgrund ihrer Kompetenz, vorsichtigeren Einschätzung oder Religionszugehörigkeit bestätigen, aber möglicherweise auch, dass diese durch

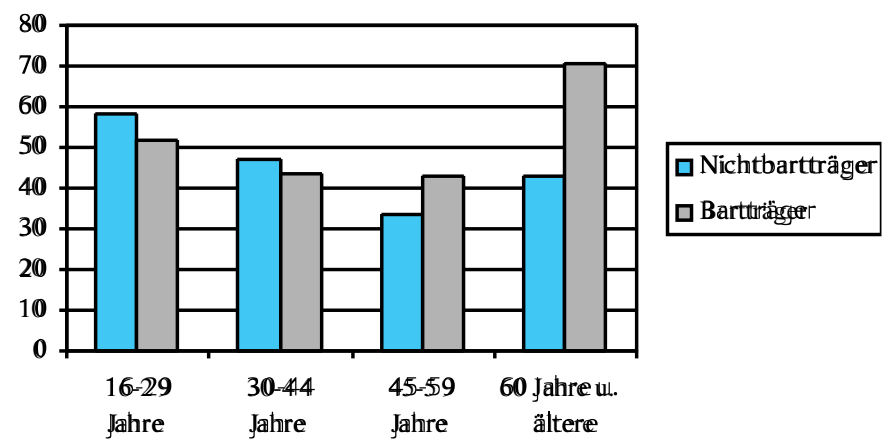

Abb. 7 Die Form des Bartes sagt etwas über Charaktereigenschaften seines Trägers aus.

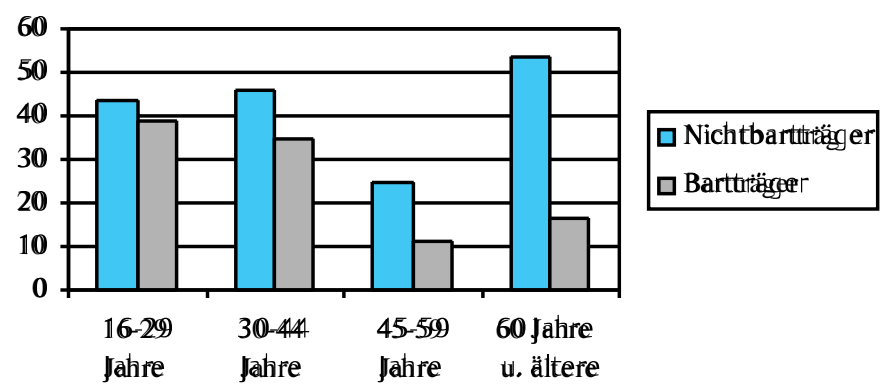

Abb. 8 Drücken lange Bärte die Zugehörigkeit zu einer Religionsgemeinschaft aus? 
ihre Bartoptik weniger als Mitglieder einer Religionsgemeinschaft interpretiert werden möchten. Auffällig ist die deutlichere Zustimmung in dieser Frage bei den 16-29-jährigen (39\%) und 30 -44-jährigen (35\%) Bartträgern. Möglicherweise sind für dieses Ergebnis politisches Interesse oder die Religionszugehörigkeit ausschlaggebend. Den Zwang zum Vollbart gab es bisher per staatlichem Gesetz nur in Afghanistan, dem islamischen Gottesstaat $[27,28]$. Die Ergebnisse deuten an, dass Nichtbartträger in relativ höherem Maße als Bartträger lange Bärte als Zeichen für die Zugehörigkeit zu einer Religionsgemeinschaft deuten.

\section{Diskussion - Interpretation der ermittelten Abbildungen von Männern mit Bart in der Werbung im Hinblick auf die gegenwärtige Bedeutung des Körpersignals Bart für die Kommunikation (II)}

Körper- und Modebewusstsein, Interesse an Lifestyle und Erotik scheinen bevorzugt mit viriler Darstellungskompetenz vereinbar zu sein wie die ermittelten Ergebnisse der Printmedienanalyse zeigen $[29,10,23,12]$. Virile Freiheit, Unabhängigkeit und Dynamik werden durch den signifikanten Vorrang des Dreitagebartes in den Werbeabbildungen aktueller Zeitschriften und Magazine vermittelt. Spiegelbildlich wird der erfolgreich an das Produkt gekoppelte Mehrwert über Emotionen vom Unbewussten zum Bewussten über die Stufen Motivationen, Einstellungen und Verhaltensweisen in den Printmedien lanciert [30]. Die ermittelte Präferenz des Dreitagebartes stimmt damit mit der in der deskriptiv ermittelten Datenanalyse überein. Das Ergebnis deutet an, dass Bartoptik für die visuelle Kommunikation Entwürfe authentischer individueller Persönlichkeitsprofile zu visualisieren vermag und daher von Bedeutung für die Kommunikation ist.

\section{Literatur}

${ }^{1}$ Schmitz I, Müller KM. Elementanalytische Untersuchungen von Tätowierungsfarbstoffen - Besteht eine potenzielle Gefährdung durch Tätowierungsfarbstoffe? JDDG 2004; 2: 350-353

2 Jedding-Gesterling M (Hrsg). Die Frisur. Eine Kulturgeschichte der Haarmode von der Antike bis zur Gegenwart veranschaulicht an Kunstobjekten der Sammlung Schwarzkopf und internationaler Museen, 1. Auflage. Wachholtz, Neumünster: 1988

${ }^{3}$ Wittenberg R. Computerunterstützte Datenanalyse, 1. Auflage. Stuttgart: Gustav Fischer, UTB Verlag, 1998

${ }^{4}$ Deutscher Studienpreis, Körberstiftung. Bodycheck - Wie viel Körper braucht der Mensch? Ergebnisse einer repräsentativen Umfrage des Instituts für Demoskopie Allensbach im Auftrag des Deutschen Studienpreises/Körber-Stiftung. Hamburg: Körber-Stiftung, 2000

${ }^{5}$ Stern/Gruner + Jahr: Dialoge III, Orientierungen in Gesellschaft, Konsum, Werbung und Lifestyle. Maschinenlesbares Codebuch ZA-Nr. 1946, Zentralarchiv für empirische Sozialforschung an der Universität zu Köln, Hamburg: 1991: 366

${ }^{6}$ Widmann M. Bart. In: Münchner Stadtmuseum (Hrsg). Anziehungskräfte, Variété de la mode 1786 - 1986. 1. Auflage. München: Carl Hanser Verlag, 1986: 46-51
${ }^{7}$ Eckes LK. Die Gesichtsbehaarung im Kontext der Biologie. Der Hautarzt 1987; 38: 224-229

${ }^{8}$ Belting H. Bild-Anthropologie, Entwürfe für eine Bildwissenschaft. 2. Auflage. München: Wilhelm Fink Verlag, 2001

${ }^{9}$ Landau T. Von Angesicht zu Angesicht. Was Gesichter verraten und was sie verbergen, 1. Auflage. Heidelberg, Berlin, Oxford: Spektrum Akademischer Verlag, 1993

${ }^{10}$ Antoni-Komar I (Hrsg). Moderne Körperlichkeit, Körper als Orte ästhetischer Erfahrung, 1. Auflage. Stuttgart, Bremen: DBV Verlag GmbH, 2001

${ }^{11}$ Haubl R. „Unter lauter Spiegelbildern ...“ Zur Kulturgeschichte des Spiegels, Bd. 2, Spätmoderne Spiegelshow. Über warenästhetische Mimikry. Consumo ergo sum, Zweckrationale Produktion irrationaler Gebrauchswertversprechen, 1. Auflage. Frankfurt a. M: Nexus, 1991

${ }^{12}$ Schneede M. Mit Haut und Haaren, Der Körper in der zeitgenössischen Kunst, 1. Auflage. Köln: Dumont, 2002

${ }^{13}$ Benthien C, Wulf C (Hrsg). Körperteile, Eine kulturelle Anatomie, 1. Auflage. Hamburg: Rowohlts Enzyklopädie, 2001

${ }^{14}$ Fuder D. Schnittstelle Schönheit. Ästhetische Chirurgie 2002; 3: 8 - 11

${ }^{15}$ Rötzer F. Posthumanistische Begehrlichkeiten. Selbstbestimmung oder Selbstzerstörung. In: Dülmen R v (Hrsg). Erfindung des Menschen. Schöpfungsträume und Körperbilder 1500-2000. 1. Auflage. Wien, Köln, Weimar: Böhlau, 1998: 609-632

${ }^{16}$ Tanner J. „Be a somebody with a body“ - Die Körpermaschinen der Arbeitsgesellschaft. In: Randow G v (Hrsg). Wie viel Körper braucht der Mensch, Standpunkte zur Debatte für den Deutschen Studienpreis. 1. Auflage. Hamburg: Edition Körber-Stiftung, 2001: 43-53

${ }^{17}$ Schlich T. Eine kurze Geschichte der Körperverbesserung. In: Randow G v (Hrsg.). Wie viel Körper braucht der Mensch, Standpunkte zur Debatte für den Deutschen Studienpreis. 1. Auflage. Hamburg: Edition Körber-Stiftung, 2001: 131 - 144

${ }^{18}$ Schelle KG. Die Geschichte des männlichen Bartes, 1. Auflage. Dortmund: Harenberg Kommunikation, Reprint der Ausgabe von 1774, 1983

${ }^{19}$ Frazer JG. Der goldene Zweig, Das Geheimnis von Glauben und Sitten der Völker, 1. Auflage. Hamburg: Rowohlts Enzyklopädie, Kulturen \& Ideen, 1989

${ }^{20}$ Hoffmann-Krayer E, Bächtold-Stäubli H. Handwörterbuch des deutschen Aberglaubens, 1. Auflage. Berlin: Walter de Gruyter, 1987

${ }^{21}$ Krumpholz-Reichel A. Haare und Psyche, Die Frisur - reine Kopfsache? Der Kult ums Haar. Psychologie Heute 2003; 7: 36 - 41

${ }^{22}$ Gnegel F. Bart ab, Zur Geschichte der Selbstrasur, 1. Auflage. Köln: Dumont, 1995

${ }^{23}$ Kreutzer D. Kauf mich, Männer in der Werbung, 3. Auflage. Berlin: Verlag für Bauwesen, 1999

${ }^{24}$ Sommer CM. Soziopsychologie der Kleidermode, 1. Auflage. Regensburg: Roderer, 1989

${ }^{25}$ Poschardt U. Anpassen, 1. Auflage. Hamburg: Rogner \& Reinhard bei Zweitausendeins, 1998

${ }^{26}$ Borrmann N. Kunst und Physiognomik, Menschendeutung und Menschendarstellung im Abendland, 1. Auflage. Köln: Dumont, 1994

${ }^{27}$ Mayr DF, Mayr KO. Von der Kunst, Locken auf Glatzen zu drehen, Eine illustrierte Kulturgeschichte der menschlichen Haarpracht, 1. Auflage. Berlin: Eichborn, 2003

${ }^{28}$ Küng H. Spurensuche, Die Weltreligionen auf dem Weg, Sonderausgabe. München, Zürich: Piper, 2001

${ }^{29}$ Wagner-Douglas I, Wippermann P. The Body is the message, Das Bild vom Körper in den Medien. Kunstforum International, Die oberflächlichen Hüllen des Selbst. Mode als ästhetisch-medialer Komplex 1998; 141: 185 - 196

${ }^{30}$ Randa-Campani S. Wunderbare Werbewelten, Marken, Macher, Mechanismen, 1. Auflage. Heidelberg: Edition Braus, 2001 